\section{¿ES FACTIBLE EL EDUPUNK EN LA FORMACIÓN UNIVERSITARIA ESPAÑOLA? HERRAMIENTAS 2.0, CONFECCIONANDO ESPACIOS DE FORMACIÓN}

\author{
Almudena García Manso \\ Eduardo Díaz Cano \\ Departamento de Ciencias Sociales, Universidad Rey Juan Carlos \\ Camino del Molino s/n 28940, Fuenlabrada, Madrid \\ almudena.manso@urjc.es y eduardo.diaz@urjc.es
}

\section{IS EDUPUNK FEASIBLE IN THE SPANISH UNIVERSITY EDUCATION? WEB 2.0 TOOLS, CREATING EDUCATIONAL SPACES}

\begin{abstract}
This paper aims to explain, from various academic learning experiences using Web 2.0 tools, the possibility, or not, of moving towards a more open, autonomous, and self-directed education, thinking about a subsequent retraining, something very required in the present-day labor market and social scene. It is intended to emphasize the educational use of collaborative ICT tools with quality criteria, and responsible use. Edupunk could be the starting point but with some fine tuning. The DIY (Do it yourself) education need supervision, mentoring, and guidance, that can be measured by educational standards at the formal universities by the optimal quality criteria.
\end{abstract}

KEY WORDS: Edupunk; EHEA; Web 2.0 tools; skills; training DIY; open social knowledge.

\section{INTRODUCCIÓN}

El panorama actual en la formación universitaria, el Espacio Europeo de Educación Superior, supone un reto que ha de ser abordado de forma eficaz y eficiente. Este escenario introduce un modelo de formación centrado en el alumno, enfatizando su capacidad de reciclaje formativo, una formación basada en el desarrollo de competencias que suponen el uso de métodos y técnicas formativas diferentes a las utilizadas en la formación tradicional/presencial. Para ello se propone la introducción de tecnologías Web 2.0 en el escenario formativo, cuyas características -las de las tecnologías Web 2.0- hacen que la formación adquiera matices cercanos a la filosofía DIY, de ahí que partiremos desde el Edupunk (Casanova, 2010) para poder desgranar las ventajas y desventajas observadas de las experiencias descritas en este trabajo.
RESUMEN: El presente trabajo pretende explicar, desde diversas experiencias formativas universitarias utilizando herramientas Web 2.0, la posibilidad o no de avanzar hacia una formación más abierta, autónoma, así como una formación centrada en la autoformación de cara a un reciclaje formativo posterior, algo muy requerido en el presente mercado laboral y escenario social. Se pretende enfatizar el uso formativo de TICs colaborativas con criterios de calidad y uso responsable. El edupunk sería el punto de partida pero con ciertos matices. La formación DIY -do it yourself o "hágalo usted mismo"- ha de tener una supervisión, tutorización y guia para que pueda estar inmersa en los circuitos de la formación universitaria reglada, con unos criterios de calidad óptimos.

PALABRAS CLAVE: Edupunk; EEES; herramientas Web 2.0; competencias; formación DIY; conocimiento social abierto.

La forma de entender el conocimiento, la formación y el aprendizaje ha cambiado en estos últimos veinte años (Uña, Hormigos y Martín, 2007). La formación continua, el reciclaje profesional y la formación autónoma y la calidad son los puntos clave del sistema formativo actual (Subirats, 2002).

Desde hace más de diez años se vienen probando, desarroIlando -a veces reinventando- nuevas metodologías, técnicas y enfoques pedagógicos con el fin de poder dar con la fórmula correcta para una adecuada adaptación a este nuevo panorama formativo y profesional. Los modelos de formación mixta b-learning y sus resultados como modelos de apoyo y soporte de actividades y estrategias de formación (Salinas, 2007) han sido sobradamente rentables. El uso de las TIC y de las Tecnologías Web 2.0 en los espacios docentes han mejorado y facilitado la incorporación de 
metodologías y técnicas pedagógicas poco adecuadas en métodos magistrales y unidireccionales, permitiendo llevar a cabo actividades que se enfocan a la construcción del conocimiento colaborativo, aprendizaje por competencias, fomento del trabajo autónomo y de calidad entre otros aspectos derivados de sus aplicaciones en la educación universitaria.

\section{Edupunk, Open Social Learning y aprendizaje INVISIBLE: RETOS EN UNA UNIVERSIDAD EN CAMBIO}

Aun concibiendo retos, pensando en los riesgos y tanteando ventajas el Edupunk y el aprendizaje social abierto -Open Social Learning- pueden servir de estrategias de adaptación a estos nuevos contextos socio/formativos -porosos, cambiantes, frágiles, etc.-, que precisarán de un sobreesfuerzo por parte de todos los miembros activos del sistema formativo/educativo universitario. Muchas de las cuestiones que consideran el Edupunk y el aprendizaje social abierto han sido recogidas en experiencias formativas dentro del sistema universitario, ahora bien, no hay que olvidar que el Edupunk o la educación expandida es una filosofía que, tal y como señalan sus principales precursores; es una filosofía de formación abierta, hecha por uno mismo, un espacio de formación que se sitúa en los márgenes del sistema institucional formativo, utilizando unos medios que se escapan del circuito institucional, enfatizando que no se pretende utilizar las tecnologías como un estándar de producción reglada, sino un medio de aprendizaje donde los alumnos son el eje central del conocimiento creado, utilizando para ello un modelo social y comunicativo entre iguales (Presley, 2011).

El manifiesto Edupunk (Schuschny, 2010) nos proporciona una visión utópica, pero en ocasiones rozando la realidad, de un escenario formativo a futuro fuera del circuito institucional. Sus propuestas nos recuerdan, en cierto modo, a la filosofía punk de los años 70. Este manifiesto se erige como un canto crítico y revolucionario, lejano a lo convencional y establecido, sin normas pero bajo un criterio de respeto, colaboración y cooperación, fruto de la filosofía de las comunidades sociales cercanas al Open source, Software libre e igualmente conexas a la filosofía de la fundación Wikimedia: creación de conocimiento compartido y distribuido, pero de calidad. Esta reflexión se puede observar en las propuestas del mismo manifiesto:
Reflexionando sobre el propio manifiesto Edupunk y comparando su filosofía y propuestas con el panorama universitario actual nos cabe pensar en una reducida aplicación de sus filosofías, siendo una aplicación parcial, limitada, tutorizada o guiada en los ciclos de grado universitario, más intensificada y abierta en los postgrados y casi plenamente incorporada en la formación continua, formación doctoral, de investigación y profesional. El Open Social Learning es una filosofía positiva, fomentadora de ideas, formas de vida, competencias y actitudes positivas tales como son la cooperación, la solidaridad, la capacidad de esfuerzo por el bien común, el intercambio de conocimientos e ideas, capacidad de respeto, entre otras actitudes (Kamenetz, 2010).

El aprendizaje invisible sería un aspecto que podria incorporarse en un futuro a estos proyectos y experiencias formativas. El conocimiento no sólo debe ser compartido y distribuido sino que debe ser abierto y accesible. Esto último permite que la filosofía Edupunk y Open Social Learning tengan éxito en su desarrollo. Sería una "nueva tecnología de aprendizaje y de oportunidades sociales... Nuestras investigaciones en el Oxford Internet Institute muestran que, aunque el acceso no se distribuye de forma homogénea en todos los grupos socioeconómicos, quienes tienen acceso a Internet acuden a la red para hacer de todo, desde buscar la definición de una palabra hasta investigar..." (Cobo, Moravec, 2011, 15).

El Edupunk y el aprendizaje invisible se apoyan en el aprendizaje social abierto y en el éxito de comunidades de usuarios que llevan utilizando el aprendizaje social abierto desde hace años (comunidades de software libre Free software comunity, open source entre otras). Estas comunidades llevan muchos años apoyándose en la confianza, cooperación y carácter emprendedor de los miembros que aportan y comparten sus conocimientos por el bien del desarrollo común. El reto y por ello la incertidumbre recae en lo sólida que es la confianza puesta en la comunidad de usuarios y en la calidad del conocimiento construido, distribuido y propagado.

El escenario de la universidad del siglo XXI ha de cambiar. Los modelos férreos y cerrados de distribución y acceso al conocimiento son modelos considerados un tanto caducos, no muy adecuados en una sociedad que ha nacido o se ha visto inmersa en un modelo de experiencias sociales y de 
aprendizaje basado en las tecnologías comunicativas de Internet. Nativos o inmigrantes digitales (Piscitelli, 2009).

Cada vez son más los que se apuntan a un modelo de apertura, distribución y compartimiento de la información y el conocimiento. El modelo Wikimedia Foundation, los modelos del entorno software libre (GNU/Linux), los blogs de contenidos pedagógicos y el uso que de las redes sociales se está haciendo, nos permiten concebir cómo estas filosofías de vida pedagógica podrían tener un éxito relativo en el sistema universitario, con matices y enfocado a ofrecer una calidad probada y testada por la institución y los docentes.

El hecho de hacer que estos modelos pedagógicos entren en escena de manera formal e institucional permiten, a nuestro entender, reforzar competencias sociales, algunas de ellas no mencionadas en los libros blancos de las titulaciones. Competencias muy necesarias en el futuro desarrollo profesional y ciudadano del alumno. Nos referimos a: la capacidad de compartir, el trabajar por un bien común, el respeto hacia la singularidad, la diversidad de opiniones y la solidaridad, entre otras.

\section{Experiencias Open Social Learning y Edupunk: DESARROLLANDO EXPERIENCIAS}

La primera de las experiencias que vamos a tratar fue llevada a cabo hace cuatro años. Se desarrolló en el marco de una asignatura cuatrimestral de primer curso de grado en Periodismo, en la asignatura Estructura social contemporánea. La experiencia partía de la realización de un blog a modo de repositorio de comentarios a textos entregados y debatidos en clase, la actividad correspondía a un $10 \%$ de la nota final y se consideraba dentro de las actividades a realizar fuera de las horas presenciales del alumno. El/la alumno/a tenía que argumentar su postura o interpretación de uno de los textos o temas debatidos en clase, los temas eran tomados de las noticias reales o de textos de libros de crítica social, el/la alumno/a debía al menos introducir una entrada en el blog durante todo el cuatrimestre para que pudiera ser considerada una actividad válida o evaluable.

Se estableció una fecha límite de inclusión de entradas en el blog y se pidió a los/as alumnos/as que se identifi- casen con un nickname facilitado a la profesora. El blog era cerrado, la inclusión de entradas estaba limitada a la comunidad de alumnos lo cual suponía un control de las entradas para facilitar su posterior evaluación. La dinámica de trabajo del alumnado se tornó en flexible, colaborativa y sobre todo se pasaba a comentar lo sucedido on-line en los debates presenciales en clase, una permeabilidad que mejoraba el clima de comunicación e intercambio de opiniones, ideas y argumentos. Lo que sucedió después del final del curso académico es lo que nos ha permitido contemplar la necesidad de estas experiencias. El blog siguió siendo mantenido por un grupo de alumnos durante casi dos años, se fueron incorporando otros usuarios -una vez terminada la experiencia y con el consentimiento de los alumnos, el blog quedo abierto a toda la comunidad de usuarios-, los comentarios y entradas eran vigiladas por la administradora del blog que era la profesora, moderando los comentarios que se incorporaban.

Aplicar una actividad formativa basada en los principios de las TIC 2.0, del open social learning, del edupunk y del aprendizaje invisible nos ha permitido contemplar que es posible socializar a un grupo de alumnos/as en estos contextos colaborativos, de intercambio y trabajo semiautónomo, desarrollando posteriormente competencias que permitan establecer hábitos de formación continua reciclaje formativo, colaboración y confianza. El hecho de que el blog siguiese activo durante un período de tiempo determinado tras finalizar su objetivo académico, nos permitió considerar la necesidad de establecer canales de conocimiento alternativos a los formales e institucionales, sin que por ello éstos sean considerados peligrosos o de riesgo para el desarrollo y la calidad de la formación Universitaria.

La segunda experiencia se llevó a cabo en el marco del proyecto de innovación docente Metodologías docentes activas a través del diseño y uso de wikis (Universidad Rey Juan Carlos). Constaba en proponer como actividad docente y evaluable (equivalente a un $35 \%$ de la nota final) la incursión por parte de los/as alumnos/as de una entrada en la wiki temática de la asignatura (Estructura social contemporánea, primer curso del Grado en Publicidad y Relaciones Públicas). El trabajo que realizaban los alumnos era un trabajo de investigación en equipos de no más de cinco miembros, cuya finalidad era la de redactar el artículo o definición extendida a incorporar en la wiki. La profesora 
tutorizaba el trabajo de investigación, facilitándoles una guía de apoyo para llevar a cabo dicha labor de manera científica y formal. Se realizaban tutorías presenciales y on-line, estas últimas en el foro de la wiki.

Respecto a los comentarios entre los equipos de trabajo sobre los artículos o definiciones extendidas, éstos, los comentarios, estaban permitidos y se consideraban necesarios. La wiki era una wiki cerrada, sólo podían incorporar contenido el supervisor/líder del equipo de trabajo. Las identidades de los supervisores/líder de equipo sólo eran conocidas por la profesora y por los miembros del equipo de trabajo, evitándose de esta forma confrontaciones entre alumnos a la hora de incluir comentarios.

Los/as alumnos/as se iniciaron en un doble aprendizaje. Por un lado tenían que aprender a utilizar una herramienta con la que estaban familiarizados pero sólo como meros usuarios y no como creadores de contenidos. Por otro lado tenían que aprender a trabajar en equipo, de forma colaborativa, de manera más autónoma que de costumbre. Tenían que hacerse a la idea de que su trabajo era constructivo y construido por todos/as, una forma de aprender que se extendía fuera del aula, pudiéndose extender en un tiempo posterior al curso académico, mostrando que todos/as trabajaban por un bien común: la wiki de la asignatura.

Los blogs y Wikis como actividades y apoyo docente se vienen utilizando desde principios de la primera década del siglo XXI (Gewerc, 2005). Estas experiencias han sido en su mayoría fructíferas, estableciendo una serie de ventajas principales, muy necesarias de ser consideradas por la metodología del aprendizaje basado en competencias, a indicar: ayudan a los alumnos a convertirse en expertos, aumentan el interés de los alumnos en el aprendizaje autónomo, incrementan el interés por la participación colectiva, ofrecen un espacio de formación abierto, fuera y anexo al aula convencional, permiten incrementar la competitividad entre los alumnos, confeccionan espacios de formación más allá de la mera formación institucional, públicos, compartidos y accesibles a toda la comunidad de usuarios, esta última ventaja se aproxima más a la filosofía del conocimiento social abierto y del aprendizaje invisible sin dejar a un lado a la filosofía DIY -do it yourself- propia del edupunk. Estas experiencias muestran como una wiki o un blog son herramientas de alfabetización tecnológica, transmisoras de valores de cooperación y colaboración, aspectos muy necesarios en el desarrollo profesional y cívico de los alumnos (Gutiérrez Martín, 2003).

\section{Resultados y conclusiones}

Estas pequeñas experiencias nos han permitido ver que sí es posible llevar a cabo actividades que se aproximan a estas filosofías y paradigmas pedagógicos. La socialización en este tipo de espacios de colaboración y construcción de conocimiento compartido y común, mediante actividades formativas permiten a los/as alumnos/as ver las ventajas de estas tecnologías-herramientas y que puedan utilizarlas provechosamente, concibiéndolas como repositorios generales de contenidos de utilidad y conocimiento hábiles en su futuro profesional en cuanto a la necesidad de estar continuamente formados.

El Edupunk y las demás filosofías indicadas en este trabajo son factibles en la universidad española de forma parcial. El Edupunk nace como una pedagogía para vivir fuera de los márgenes de la institucionalidad de la formación universitaria (Ebner, 2008). Aun así, sería posible aplicar algunos de sus rasgos o características, a enumerar: el aprendizaje mediante experiencias, conscientes e inconscientes, que han acontecido en cualquier momento o sitio y no sólo en los circuitos convencionales e institucionales -algo que acontece en cualquier interacción comunicativa en blogs, wikis y redes sociales- y que pueden ser paralelos o de apoyo a los mismos.

La capacidad de desarrollar un contenido y conocimiento de forma autónoma -do it yourself- desarrollada por medios propios. La ruptura entre el antagonismo real/virtual, las experiencias utilizadas en este trabajo y todas aquéIlas que están basadas en las tecnologías Web 2.0, son experiencias b-learning o e-learning y por ello partícipes del mundo de lo digital. Son experiencias de apoyo a la docencia, a lo real, de lo que se deduce que no existe antagonismo alguno, que los "hechos" acontecidos en lo digital infieren en lo real. Un texto trabajado en una wiki aporta conocimiento real. Investigar a través de la acción, en las wikis y en los blogs el hecho de introducir un comentario de calidad infiere investigar mediante la acción. Las clases son conversaciones, en estas experiencias la participación del docente es a modo de conversación multidireccional 
con los alumnos, ellos son los que crean el contenido, el docente es quien dialoga y conversa sobre los aspectos relevantes de sus trabajos. El edupunk no es lo que pasa en el aula sino que es el mundo en el aula. Las herramientas Web 2.0 mantienen la actividad formativa fuera del aula convencional pero incluyen de manera más dinámica temas más amplios y variados -ejemplo de ello lo encontramos en los foros de la wiki, donde los alumnos comentaban noticias relacionadas con sus temas a desarrollar-. Sentirse parte de un trabajo colectivo y hacer ver que sin colaboración la educación es una ficción, hacer que los alumnos asuman su rol colectivo, su responsabilidad en el trabajo común, su presencia como miembro del colectivo que crea el conocimiento para los demás. Hágalo usted mismo pero también y esencialmente hágalo con otros.

Muchas son las ventajas que de estas filosofías pedagógicas se podrían obtener, pero la realidad nos muestra que sólo pueden ser incluidas de manera parcial, como pequeñas cápsulas de aire fresco que agilizan el sistema formativo, permitiendo en un futuro que la universidad pueda crear cauces de conocimiento abierto, colaborativo y al alcance de toda la comunidad de usuarios y en continuo reciclaje.

\section{BIBLIOGRAFÍA}

Alemán, A. (2010): "Una crítica al sistema educativo español", en Derecho y Educación, en P. Martínez Ruano (coord.), Universidad de Alcalá de Henares, Alcalá de Henares, pp. 43-58.

Casanova, D. (2010): "Punking up Education! New perspectives for teaching and learning", Revista Indagatio Didactica, vol. 2, 1, pp. 85-93.

Cobo, C. y Moravec, J. W. (2011): "Aprendizaje invisible. Hacia una nueva ecología de la educación", Colleció Transmedia XXI, Laboratori de Mijants Interactius, Barcelona, Publicacions i Edicions de la Universitat de Barcelona, p. 15.

Ebner, M. (2008): "Why we need edupunk", Revista de Informatica Sociala, vol. 9, pp. 32-40.

García Manso, A.; Díaz Cano, E. y Martín Cabello, A. (2011): "Experiencia: método docente activo de aprendizaje basado en competencias a través del diseño y uso de wikis", Boletín de la ETSII, n. ${ }^{\circ}$ 1, pp. 19-24.

Gewerc Barujel, A. (2005): "El uso de weblogs en la docencia universitaria", Revista Latinoamericana de Tecnología Educativa, vol. 4, 1, pp. 9-24.
Gutiérrez Martín, A. (2003): Alfabetización digital: algo más que ratones y teclas, Barcelona, Gedisa.

Kamenetz, A. (2010): DIYU. Edupunks, Edupreneurs, and the Coming Transformation of Higher Education, Vermont, Chelsea Green Publishing Company.

Piscitelli, A. (2009): Nativos digitales. Dieta cognitiva, inteligencia colectiva y arquitecturas de participación, Buenos Aires, Santillana.

Presley, J. W. (2011): "DIY U: Edupunks, Edupreneurs, and the Coming Transformation of Education", The Review of Higher Education, vol. 34, 4, pp. 714-717.

Salinas, J. (2007): "Innovación docente y uso de las TIC en la enseñanza Universitaria", Revista Universidad y Sociedad del Conocimiento, vol. 1, 1, pp. 1-16.

Schuschny, A: Manifiesto edupunk [acceso 13/09/2010]: http://www.edupunkmanifesto. org/manifiesto-edupunk/

Subirats, J. (2001): "Universidad en España: ¿época de cambios o cambio de época?", Educar, 28, pp. 11-39.

Uña, 0.; Hormigos, J. y Martín, A. (2007): Las dimensiones sociales de la globalización, Madrid, Paraninfo. 